\title{
THE EFFECTS OF SLEEP APNEA ON HEART RATE VARIABILITY IN A SIMULATOR
}

\author{
Linda Boyle, ${ }^{1,3}$ John Hill, ${ }^{1}$ Jon Tippin, ${ }^{2}$ Kevin Faber, ${ }^{2}$ Matthew Rizzo ${ }^{1,2,3}$ \\ ${ }^{1}$ College of Engineering \\ ${ }^{2}$ College of Medicine \\ ${ }^{3}$ Public Policy Center \\ University of Iowa, Iowa City, Iowa, USA \\ E-mail: linda-boyle@uiowa.edu
}

\begin{abstract}
Summary: Heart rate variability has been used as a measure of mental workload, stress, and fatigue in drivers. The main goal of this study was to evaluate whether drivers with obstructive sleep apnea syndrome (OSAS) may have significantly different heart rate variability from those who do not have OSAS. Such a condition may indicate lower stress levels and an increase in crash risk due to sleepiness. This study also evaluates whether significant deviations in HRV may occur as drivers become drowsier over time. Eleven drivers with OSAS were compared to twelve other drivers with no known sleep disorder. All were tested in a driving simulator over a 60 -minute period that consisted of three uneventful drive segments on two-lane rural roads. Heart rates were collected using electrocardiogram (ECG). The variability of heart rate was computed for each subsequent five-minute interval by calculating the standard deviations of the R-R intervals (i.e., the time duration between two consecutive R waves of the ECG) within that time. Results showed that there were no significant differences in HRV over time for the comparison group. However, HRV for drivers with OSAS increased by each subsequent time interval. Drivers with OSAS also had significantly higher mean heart rate variability over the course of the drive. Specifically, based on the second regression model, the difference in heart rate variability between drivers with OSAS and the comparison group significantly increased after about 25 minutes of driving. This likely reflects the physiological effects of increased fatigue, which would lead to inattention to the driving environment and increased crash risk.
\end{abstract}

\section{INTRODUCTION}

Driver sleepiness is a contributing factor of motor vehicle crashes (Horne \& Reyner, 1995; Laube et al., 1998) and may be attributed to driver fatigue due to increased workload or medical conditions such as Obstructive Sleep Apnea Syndrome (OSAS). OSAS is a common sleep disorder that affects 2 to $4 \%$ of middle-aged adults (Young et al., 1993). Drivers with OSAS have an increased crash risk that can be up to 4 times that of the general population (TeranSantos et al., 1999; Wu \& Yan-Go, 1996). Past research has also shown that this particular sleep disorder can result in an inability to maintain safe driving performance, particularly during microsleep episodes (Paul, Boyle, Boer, Tippin \& Rizzo, 2005).

Heart rate variability (HRV) provides a means to quantify drowsiness physiologically (Mulder, 1992), and is defined as the beat to beat variations in heart rate (i.e., the R-R intervals). This measure has been previously used to examine mental workload (Hancock and Verwey, 1997), 
stress (Bornas et al., 2004; Steyvers and DeWaard, 2000), and driver fatigue (Egelund, 1982). With respect to driver fatigue, or drowsiness, HRV measures can provide insights related to the time in the driving task when fatigue becomes problematic. An increase in HRV is typically an indication of decreases in mental workload, which can occur in sleepy drivers over prolonged monotonous driving. Although lower workload and stress is typically better, they may also relate to lower vigilance, which can negatively impact driver performance (Horne and Reyner, 1995).

Heart rate variability (HRV) can be evaluated using time-based or frequency measures. The time domain is more common and can be assessed with straightforward calculation of the standard deviation of R-R (inter-beat) intervals. Using this technique, Wood et al. (2002) examined HRV among elderly and young participants while they performed demanding visual and auditory tasks. They found that HRV was significantly lower among elderly participants. Spectral or frequency domain analysis is based on mathematical transformations (i.e., Fourier transforms) of the signal from time to frequency (expressed in cycles per beat with varying amplitudes and frequencies). This method has been used to assess the component of HRV associated with pulmonary control (Mulder, 1992), posttraumatic stress disorder (Knorr et al., 2003), and mental workload (Richter et al., 1998). In the latter study, spectral analysis was conducted on data from an on-road study of rural driving to examine decreases in $0.1 \mathrm{~Hz} \mathrm{HRV}$ amplitude during more difficult road segments (Richter et al., 1998). They observed that drivers had lower HRV on an easier road segment immediately after a difficult road segment.

The advantage of examining HRV in the time domain is the simple computation methods and the ability to assess instantaneous HRV changes. The advantage of the frequency domain is the ability to separate components of HRV (low-frequency power vs. high-frequency power). The broad goal of this study is to assess physiologic indices of performance in drivers who are at risk for driving safety errors due to fatigue associated with OSAS. This study evaluates the hypothesis that sleepy drivers with obstructive sleep apnea syndrome (OSAS) have greater heart rate variability (HRV) during driving compared to drivers who do not have OSAS. The outcomes are evaluated with respect to time and spectral analysis.

\section{METHODOLOGY}

\section{Participants}

Eleven drivers who met clinical criteria for OSAS (age: $M=45.64 \mathrm{SD}=8.67)$ (AASM, 2005) were compared to twelve drivers who did not have evidence of a sleep disorder (age: $M=45.00$ $\mathrm{SD}=7.78$ ). None of the drivers with OSAS had received treatment for their condition prior to the study. Drivers were also paid $\$ 50$ for their participation.

\section{Experimental Procedure}

All driver participants were tested in driving scenarios implemented on SIREN: Simulator for Interdisciplinary Research in Ergonomics and Neuroscience (Rizzo et al., 2000). Each participant drove on three laps of a simulated 2-lane highway with minimal traffic to induce sleepiness. Each lap consisted of curved road segments with 600-meter radii (3 left turn, 3 right turn). 
Participants were instructed to drive at speeds below the 55-mph speed limit and it took approximately 60 minutes to complete a test.

\section{Performance Measures}

Heart rates in all drivers were assessed using electrocardiography (ECG) (Neurofax, Nihon Kohden, Tokyo). The response variable analyzed in this study was heart rate variability (HRV), and this was computed from ECG data using two methods. For the time domain, HRV was determined for each consecutive five-minute segment of the drive by calculating the standard deviations of time between the R-R intervals (i.e., the latency between two consecutive $\mathrm{R}$ waves of the ECG). This interval period is consistent with research conducted by Roche et al. (1999), Bonnet et al. (1998), and as described in the review of heart rate variability by van RavenswaaijArts et al. (1993). The same consecutive five-minute segments were converted to the frequency domain using a fast Fourier transform (FFT) for the spectral analysis.

For the time domain, the independent variables included driver group (OSAS, normal), and time segment (from the first 5-min. segment to the $14^{\text {th }}$ five-minute segment). A couple of participants had 15 and 16 time segments. However, these segments were not included due to the small samples.

\section{Statistical Analysis}

Time domain analysis: Inter-beat intervals and HRV were calculated using Matlab 7.1 with a normalized HRV as the dependent measure (assessed on a continuous scale). This value was computed as the HRV for a given 5-minute interval divided by the HRV associated with the initial 5-minute interval. This enabled a between-subject assessment of the HRV changes over time. The time domain analysis was conducted using analysis of variance (ANOVA) techniques for repeated measures to determine whether differences in mean HRV exists over each consecutive time segment (within-subject factor) for each driver group (between-subject factor). The driver group consisted of OSAS and non-OSAS drivers (2 levels). Statistically significant interactions were then evaluated in a second model created to determine the time segment that the mean HRV began to significantly differ between driver group.

The PROC MIXED procedure in SAS 9.1 with repeated measures was used to develop the models. Regression equations for each driver group were also developed with PROC MIXED using the 'solution' and 'noint' option in SAS.

Frequency domain analysis: HRV was assessed by comparing low-frequency $(.05-.15 \mathrm{~Hz})$ to high-frequency (.15 to $.40 \mathrm{~Hz}$ ) power using a fast Fourier transform (FFT) of the same 5-minute segments used in the time domain. This is the standard nonparametric technique for spectral analysis of HRV (Pichon et al., 2006). This analysis was conducted using HRV analysis software v.1.1 developed by Biomedical Signal Analysis Group, Department of Applie Physics, University of Kuiopio (Niskanen et al., 2002). The ratio of low-frequency (LF) to high-frequency (HF) power within the spectrum was used as the dependent variable (Pichon et al., 2006). Decreases in the LF/HF state has been previously related to a more drowsy state (Byeon et al., 2006), while increases in LF/HF tend to indicate greater mental workload or alertness (Sato et 
al., 1998). These values were plotted across all eleven OSAS participants to determine whether a trend similar to the time domain analysis could be observed.

\section{DATA ANALYSIS AND RESULTS}

Time domain: There was a significant interaction effect between consecutive time periods and driver group $(\mathrm{F}(1,268)=3.92, \mathrm{p}=0.048)$. When OSAS drivers were plotted separately from the comparison (or non-OSAS) drivers (Figure 1), the normalized HRV is observed to increase over time for the drivers with OSAS, while remaining fairly constant for the comparison group. Although there is no statistically significant difference between the comparison and OSAS groups within a single time period, the regression model demonstrates the significant increase of mean HRV over time.

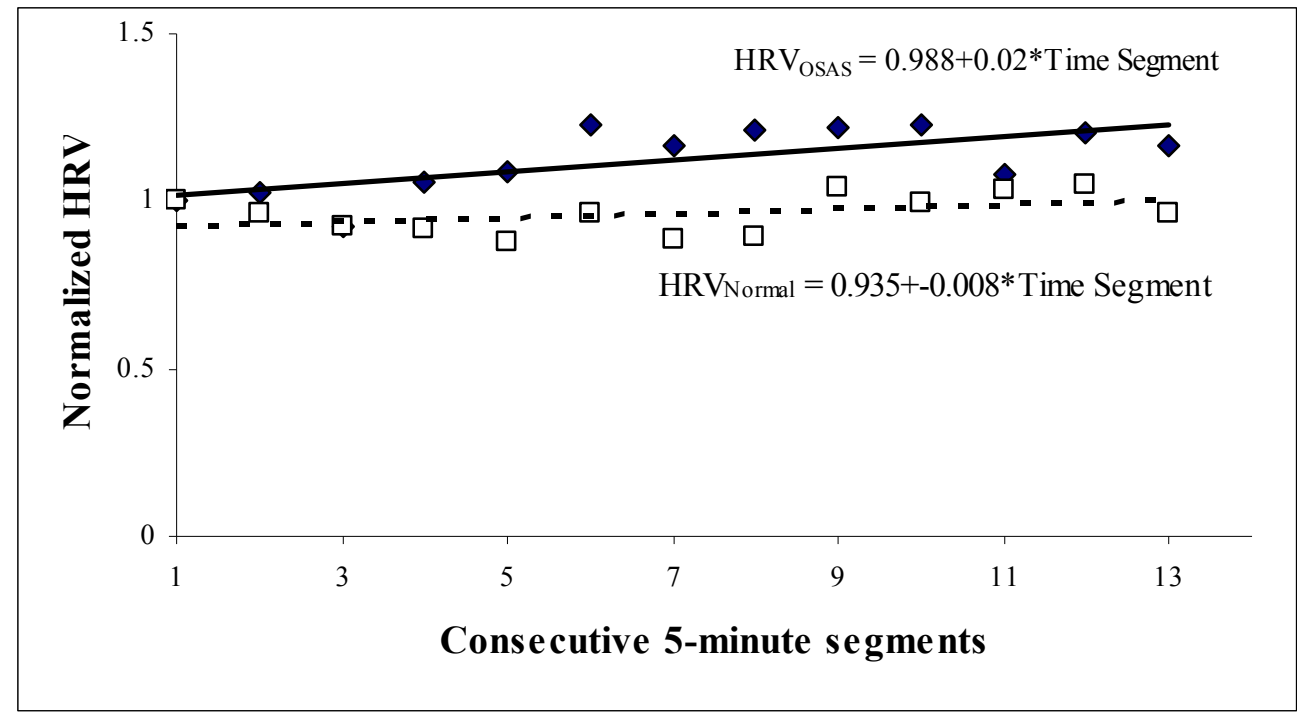

Figure 1. A comparison of normalized HRV for drivers with and without OSAS

A regression model was developed to evaluate the time period that HRV began to differ significantly between OSAS and non-OSAS drivers. The analysis revealed that the differences in mean HRV began after 25 minutes of driving (or after the $3^{\text {rd }}$ consecutive 5 -minute segment) $(\mathrm{p}<0.001)$ (eq. 1). This difference was a result of the interaction between elapsed time (or extended driving) and the presence of OSAS.

$$
H R V=0.983+0.189(O S A S)+0.150(O S A S \times \text { ExtendedDriving }) \text { for } \mathrm{n}=157
$$

Frequency domain: The power spectral density is represented by the ratio of low frequency (LF) to high frequency (HF). Based on the results from the time domain analysis, LF/HF values were expected to decrease over time. However, as seen in Figure 2, there is actually no significant change in HF/LF with each consecutive time interval. 


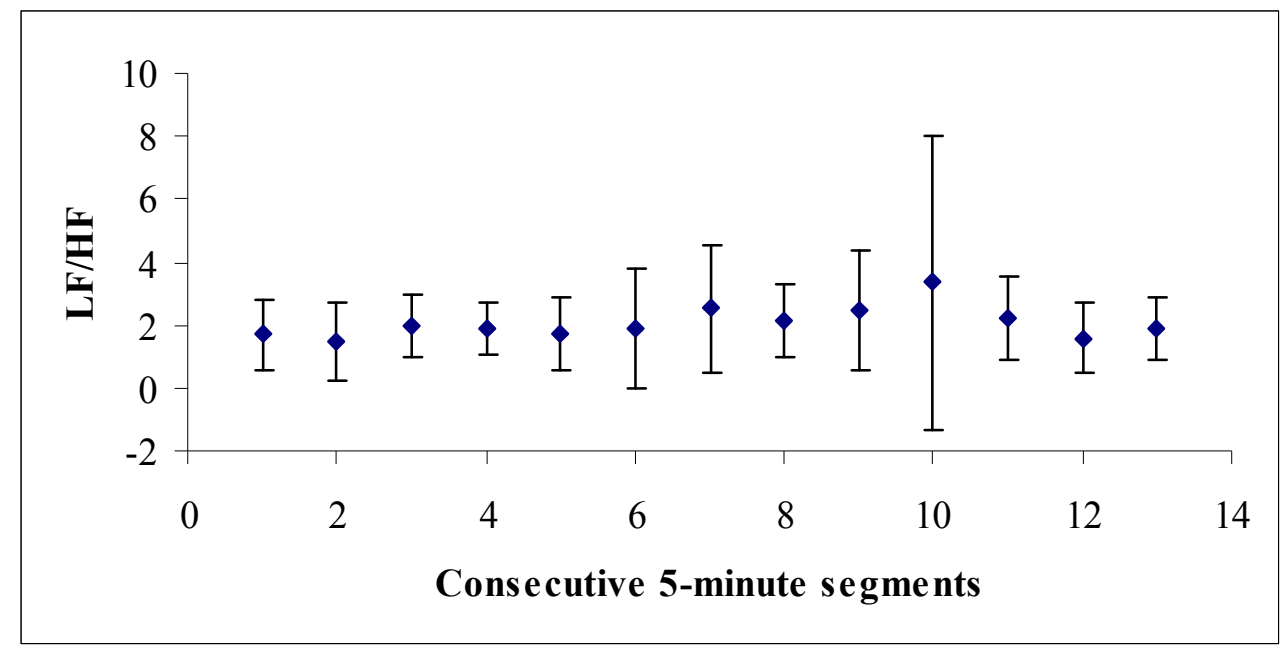

Figure 2. Plot of LF/HF ratio over each consecutive drive segment

(error bars represent one standard deviation)

\section{DISCUSSION}

This study examined the use of HRV for detecting drowsiness in drivers. Using the time domain, HRV significantly increased during simulated driving in OSAS drivers when compared to nonOSAS drivers. Both driver groups had similar HRV at the onset of the drive. However, after prolonged monotonous driving, HRV started to increase for drivers with OSAS, indicating a greater drowsiness state. This is consistent with other studies that used the time domain to examine sleepiness (Roche et al., 1999; van den Berg et al., 2005). The increase in mean HRV observed in the time domain also parallels increased self-ratings of sleepiness, and declines in driving control and performance on a vigilance task in drivers with OSAS (Tippin et al., 2007). These sleepy drivers are less aware of their surroundings and pay less attention to the driving task and environment.

Spectral analysis provides the ability to distinguish between parasympathetic (HF) and sympathetic (LF) activity with LF/HF being the sympathovagal balance. Interestingly, the spectral analysis did not yield significant results. This may be due to noise generated within the data, which does not allow for the identification of all intervals. As a result, for some subjects, the Fourier transformation removed some intervals when the signal could not be discerned from the noise. This reduced the ability of the spectral analysis to identify the LF part of the overall signal. This is seen in the variability within the LF segment seen in Figure 3. This does not affect the temporal analysis, because each interval is treated as a separate outcome. As a result, the few missing data points could be assumed to follow the same sample distribution and not affect the standard deviation measure for that time period. However, missing intervals have a significant effect on the spectral analysis, particularly when determining the LF component. Although both time and frequency based have been widely used, the time domain has been shown to represent an accurate and inexpensive screening tool for clinically suspected OSAS patients (Roche et al., 1999) and appears promising for participants in our study as well. However, further analysis is needed with additional subjects to validate this outcome. 


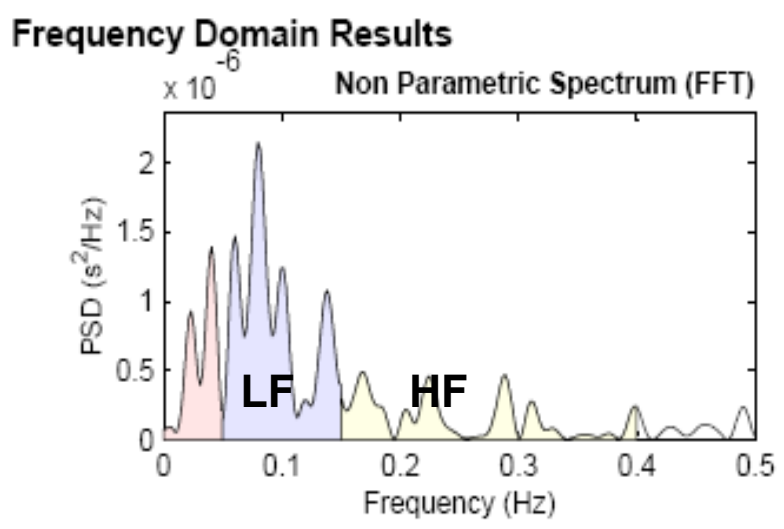

Figure 3. Frequency domain results for one subject

The findings of this study suggest that HRV may provide another measurable physiologic signature of drowsy driving that can add to the profile of quantifiable physiologic changes in sleepy drivers. Development of in-vehicle alerting and warning devices activated by changes in HRV may help mitigate the deleterious effects of decreased alertness upon driver performance.

Analyzing HRV over time is effective in not only comparing physiological measures between OSAS and comparison groups, but also in determining when HRV may significantly reduce the awareness needed by the driver to attend to driving. The time-dependency of this effect is important because it allows possible counter-measures for fatigue to be assessed for their longterm effectiveness. This is also important for determining guidelines for trip duration and other constraints designed to make driving safer.

\section{REFERENCES}

American Academy of Sleep Medicine (AASM). (2005). International classification of sleep disorders, 2nd edition: Diagnostic and coding manual. Westchester, Illinois: American Academy of Sleep Medicine, 51-55.

Bonnet, M., and Arand, D. (1998) Heart rate variability in insomniacs and matched normal sleepers. Psychosomatic Medicine, 60, 610-615.

Bornas, X., Llabres, J., Noguera, M., Lopez, A., Barcelo, F., Tortella-Feliu, M., et al. (2004). Self-implication and heart rate variability during simulated exposure to flight-related stimuli. Anxiety, Stress, and Coping, 17(4), 331-339.

Byeon, M., Han, S. Min, H., Wo, Y., Park, Y. (2006). A study of HRV analysis to detect drowsiness states of drivers. International Association of Science And Technology For Development, Proceedings of the 24th IASTED international conference on Biomedical engineering, 153-155.

Egelund, N. (1982). Spectral analysis of heart rate variability as an indicator of driver fatigue. Ergonomics, 25, 7, 663-672.

Hancock, P., \& Verwey, W. (1997). Fatigue, workload and adaptive driver systems. Accident Analysis and Prevention, 29(4), 495-506. 
Horne, J.A., \& Reyner, L.A. (1995). Sleep related vehicle accidents. British Medical Journal, $310(6979), 565-567$.

Knorr, B., Akay, M., and Mellman, T. (2003) Heart rate variability during sleep and the development of PTSD following traumatic injury. Proceedings of the $25^{\text {th }}$ Annual International Conference of the IEEE EMBS, Cancun, Mexico, 354-357.

Laube, I., Seeger, R., Russi, E.W., \& Bloch, K.E. (1998). Accidents related to sleepiness: review of medical causes and prevention with special reference to Switzerland. Schweiz Med Wochenschr, 128(40), 1487-1499.

Mulder, L. (1992). Measurement and analysis methods of heart rate and respiration for use in applied environments. Biological Psychology, 34, 205-336.

Niskanen, J., Tarvaienen, M., Ranta-aho, P, and Karjalaienen, P. (2002) Software for advanced HRV analysis. ISSN 0788-4672. Accessed on Nov. 25, 2006, at http://bsamig. uku.fi/winhrv.shtml

Paul, A., Boyle, L., Tippin, J., \& Rizzo, M. (2005). Variability of driving performance during microsleeps. Proceedings of the 3rd International Driving Symposium on Human Factors in Driving Assessment, Training, and Vehicle Design. Iowa City, IA: Public Policy Center, University of Iowa, 433-440.

Pichon, A., Roulaud, M., Antoine-Jonville, S., Bisschop, C., Denjean, A. (2006) Spectral analysis of heart rate variability: interchangeability between autoregressive analysis and fast Fourier transform. Journal of Electrocardiology, 39, 31-37.

Richter, P., Wagner, T., Heger, R., \& Weise, G. (1998). Psychophysiological analysis of mental load during driving on rural roads - a quasi-experimental field study. Ergonomics, 41(5), 593-609.

Rizzo, M., McGehee, D., \& Jermeland, J. (2000). Design and installation of a driving simulator in a hospital environment. In K. Brookhuis, D. DeWaard \& C. Weikert (Eds.), Human System Interaction: Education, Research and Application in the 21st Century. Maastricht, The Netherlands: Shaker Publishing, 69-77.

Roche, F., Gaspoz, J., Court-Fortune, I., Minini, P., Pichot, V., Duverney, D., Costes, F., Lacour, J. and Barthelemy, J. (1999) Screening of Obstructive Sleep Apnea Syndrome by Heart Rate Variability Analysis, Circulation, 100, 1411-1415.

Sato, N., Kamada, T., Miyake, S., Kumashiro, M, and Kume, Y. (1998) Power spectral analysis of heart rate variability in type A females during a psychomotor task. Journal of Psychosom Research, 45(2), 159-169.

Steyvers, F., \& DeWaard, D. (2000). Road Edge delineation in rural areas: effects on driving behaviour. Ergonomics, 43(2), 223-238.

Teran-Santos, J., Jiminez-Gomez, A., \& Cordero-Guevara, J. (1999). The association between sleep apnea and the risk of traffic accidents. The New England Journal of Medicine, 340(11), 847-851.

Tippin, J., Rizzo, M., Sparks, J.D., Boyle, L.N. (2007). Visual Vigilance in Drivers with Obstructive Sleep Apnea Syndrome (OSAS). 59th Annual Meeting, American Academy of Neurology, Boston, Massachusetts, April 28-May 5. 
Van Ravenswaaij-Arts C., Kollee, L., Hopman, J., Stoelinga, G., and van Geijn, H. (1993) Heart rate variability. Annals of Internal Medicine, 118(6), 436-447.

Van den Berg, J., Neely, G., Wiklund, U., Landstrom, U. (2005) Heart rate variability during sedentary work and sleep in normal and sleep-deprived states. Clinical Physiol Funct Imaging, 25, 51-57.

Wood, R., Maraj, B., Lee, C., Reyes, R. (2002) Short-term heart rate variability during a cognitive challenge in young and older adults, Age and Ageing, 3, 131-135.

Wu, H., \& Yan-Go, F. (1996). Self-reported automobile accidents involving patients with obstructive sleep apnea. Neurology, 46(1254-1257).

Young, T., Palta, M., Dempsey, J., Skatrud, J., Weber, S., \& Badr, S. (1993). The occurrence of sleep-disordered breathing among middle-aged adults. The New England Journal of Medicine, (328), 1230-1235. 